\title{
Common strength and localization of spontaneous and evoked synaptic vesicle release sites
}

Kristina Loy ${ }^{1,2 \dagger}$, Oliver Welzel ${ }^{1 \dagger}$, Johannes Kornhuber ${ }^{1}$ and Teja W Groemer ${ }^{1 *}$

\begin{abstract}
Background: Different pools and functions have recently been attributed to spontaneous and evoked vesicle release. Despite the well-established function of evoked release, the neuronal information transmission, the origin as well as the function of spontaneously fusing synaptic vesicles have remained elusive. Recently spontaneous release was found to e.g. regulate postsynaptic protein synthesis or has been linked to depressive disorder. Nevertheless the strength and cellular localization of this release form was neglected so far, which are both essential parameters in neuronal information processing.

Findings: Here we show that the complete recycling pool can be turned over by spontaneous trafficking and that spontaneous fusion rates critically depend on the neuronal localization of the releasing synapse. Thereby, the distribution equals that of evoked release so that both findings demonstrate a uniform regulation of these fusion modes.

Conclusions: In contrast to recent works, our results strengthen the assumption that identical vesicles are used for evoked and spontaneous release and extended the knowledge about spontaneous fusion with respect to its amount and cellular localization. Therefore our data supported the hypothesis of a regulatory role of spontaneous release in neuronal outgrowth and plasticity as neurites secrete neurotransmitters to initiate process outgrowth of a possible postsynaptic neuron to form a new synaptic connection.
\end{abstract}

\section{Findings \\ Background}

Central neurons display two different forms of vesicle release: stimulation dependent, i.e. evoked release and stimulation independent spontaneous release, which occurs at resting membrane potential. Spontaneous neurotransmitter release is thought to play a crucial role in synaptic plasticity, memory and learning [1] as well as in pathophysiology [2]. Furthermore spontaneous neurotransmitter release was found to regulate postsynaptic dendritic protein synthesis $[3,4]$. The origin of spontaneous release remains controversial, with some evidence arguing for the recycling pool $[5,6]$ of vesicles and some for the reserve pool [7]. Here we seek to analyze the amount of spontaneous release regarding its turnover kinetics and cellular location. While a pool can be

\footnotetext{
*Correspondence: teja.groemer@uk-erlangen.de

${ }^{\dagger}$ Equal contributors

'Department of Psychiatry and Psychotherapy, Friedrich-Alexander-University of Erlangen-Nuremberg, Schwabachanlage 6, Erlangen 91054, Germany Full list of author information is available at the end of the article
}

defined for each form of release, our results indicate a common regulation of both vesicle populations and thus a common origin of both pools from the recycling pool of vesicles.

\section{Results}

Using a live-cell imaging approach with the styryl dye FM1-43 we determined the amount of spontaneous and evoked release at individual hippocampal synapses. Therefore synapses underwent a spontaneous and an evoked round of staining, each followed by activity induced destaining (Figure 1A). For spontaneous staining incubation times of 5-240 minutes where used, depending on when saturation was reached. Calcium concentrations ranged from the total absence of calcium in the extracellular medium, physiological calcium concentrations $(2.5 \mathrm{mM})$ to a high calcium concentration $(5 \mathrm{mM})$. Using this paradigm we were able to quantify the recycling pool size as well as the spontaneously released vesicles after different loading times for spontaneously

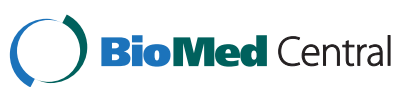




\section{A}

\begin{tabular}{|c|c|c|c|c|c|}
\hline $5-240 \mathrm{~min}$ & & & & & \\
\hline spontaneous & & & evoked & & ||||||||| \\
\hline FM 1-43 & wash & destain & FM 1-43 & wash & destain \\
\hline & & image & & & image \\
\hline $0-5 \mathrm{mM} \mathrm{Ca}^{2+}, \mathrm{TX}$ & & & $2.5 \mathrm{Ca}^{2+}$ & & \\
\hline
\end{tabular}

B
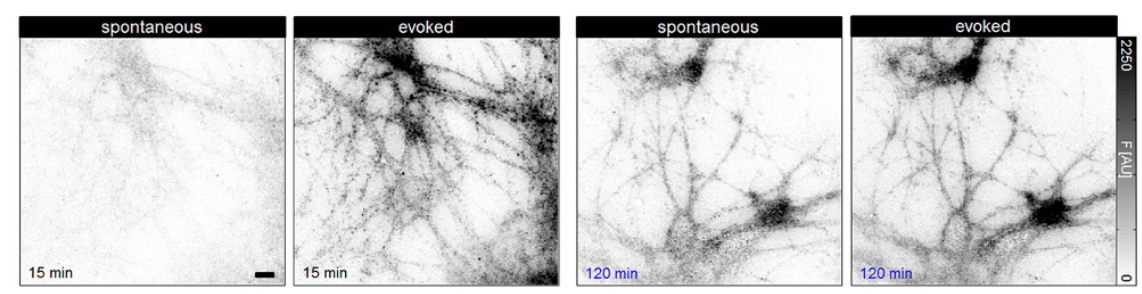

C

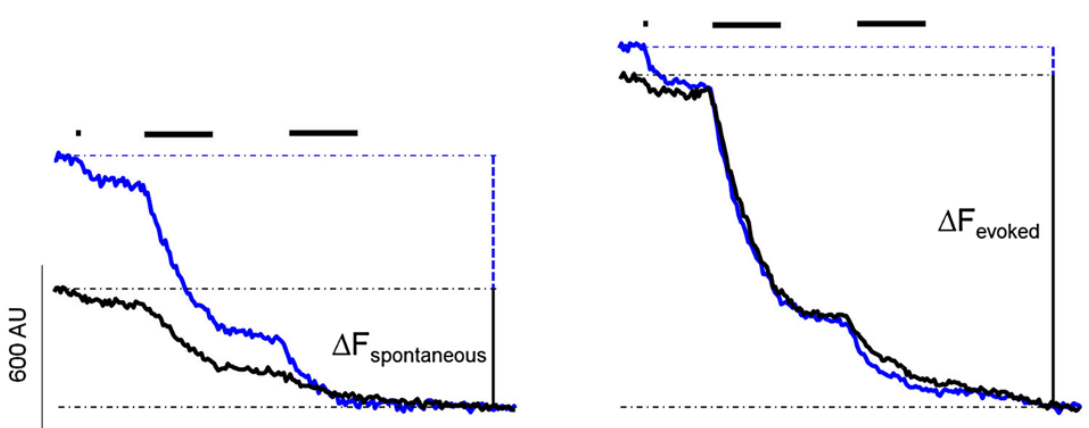

$40 \mathrm{~s}$

D

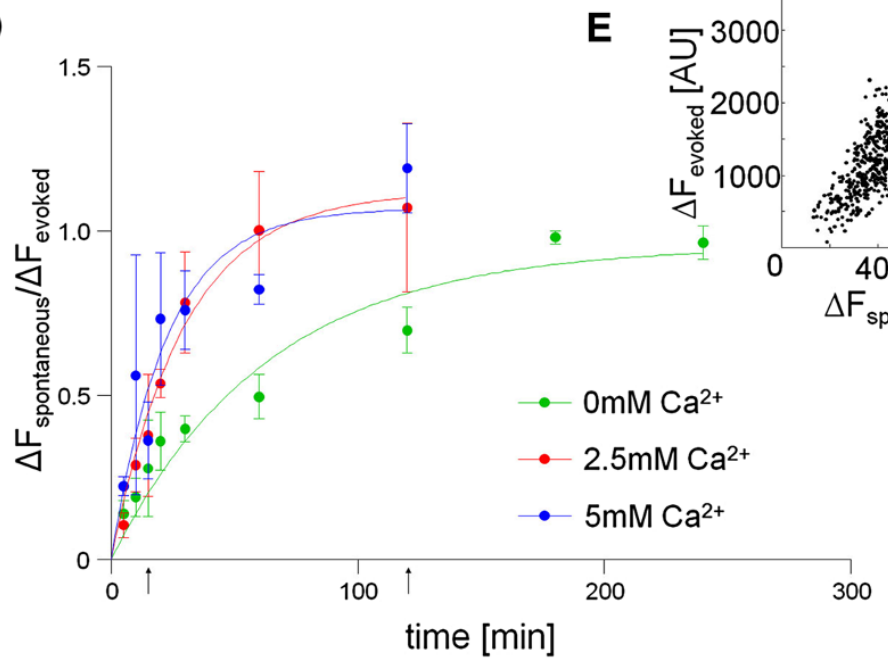

Figure 1 The recycling pool of hippocampal synapses is turned over completely by spontaneous vesicle recycling. A Scheme of experimental setup: Boutons were first labeled with FM1-43 by spontaneous uptake for different time periods. At the end of the first part of the experiment, boutons were completely destained using twice 900 pulses to determine the amount of spontaneous turnover. After a 10 minute recovery synaptic boutons were labeled a second time using 1200 electrically evoked action potentials and again completely destained to determine the recycling pool size. B Difference images for 15 and 120 minutes spontaneous FM1-43 uptake and evoked staining, respectively. Scale bar, $10 \mu \mathrm{m}$. C Corresponding mean fluorescence profiles to determine $\Delta \mathrm{F}_{\text {spontaneous }}$ and $\Delta \mathrm{F}_{\text {evoked }}$ for each time period. D Time course of $\Delta \mathrm{F}_{\text {spontaneous }}$ to $\Delta \mathrm{F}_{\text {evoked }}$ ratio depending on extracellular calcium concentration $\left(t_{1 / 2}\right.$ in minutes: $0 \mathrm{mM} \mathrm{Ca}^{2+}=43.96,2.5 \mathrm{mM} \mathrm{Ca}{ }^{2+}=20.58,5 \mathrm{mM}$ $\mathrm{Ca}^{2+}=15.73$ ). Arrows mark the exemplary time points. E Correlation of spontaneous and evoked release at individual synaptic boutons (time point at $60 \mathrm{~min})$. 
fusing vesicles. Additionally different calcium concentrations for spontaneous loading were tested. First we found that spontaneous release was able to turn over the complete recycling pool in a calcium dependent manner (Figure 1). Although the kinetics to reach saturation was slower at reduced calcium concentrations, all recycling vesicles were labeled at different concentrations of external calcium [8] (Figure 1D). Importantly [9], synapses that were capable of spontaneous vesicle release also underwent evoked vesicle recycling $(97.87 \% \pm 3.09 \%$ of boutons) and vice versa $(98.61 \% \pm 0.96 \%$ of boutons; Wilcoxon rank sum test: $p=0.83$ ) and their spontaneous and evoked fluorescence changes were correlated (Figure 1E; Pearson's $r=0.79 \pm 0.06 ; 3$ experiments, 921 boutons). To validate the turnover of the whole recycling pool by spontaneous release we used an alternative dual-color imaging approach with synapto-pHluorin $(\mathrm{spH})$ transfected neurons and an $\alpha$ GFP-CypHer5 $\mathrm{E}^{\mathrm{ma}}$ antibody to label the spontaneously fused vesicles. As both fluorescence markers were $\mathrm{pH}$-sensitive with an inverse characteristic [10,11], which made them excellent tools for the analysis of exo- and endocytosis due to the acidic $\mathrm{pH}$ inside synaptic vesicles. In contrast to the previous FM1-43 experiments evoked and spontaneous release was measured simultaneously by quantifying the fluorescence decay of the $\alpha$ GFPCypHer5 $\mathrm{E}^{\mathrm{rm}}$ fluorescence signal, resembling the spontaneous labeled vesicles, and on the other hand the $\mathrm{spH}$ fluorescence increase, corresponding to activity dependent recycling pool of vesicles. In accordance to the FM1-43 experiments we demonstrated that the whole recycling pool, which corresponds to $37.10 \pm 11.10 \%$ of the total pool, could be labeled (Additional file 1: Figure S1). In a next step we wanted to determine the fusion rate of a single spontaneously released vesicle to estimate the time to turn over the whole recycling pool of vesicles and thus to proof the kinetics described above. Therefore synapses were stained spontaneously with FM1-43 for only $15 \mathrm{mi}$ nutes at zero calcium to label only a few vesicles and subsequently destained to monitor the resulting fluorescence decay (Additional file 1: Figure S2A). Analysis of these fluorescence changes using histograms exhibited a clear quantization that corresponds to intensity of a single vesicle, which enabled us to calculate the number of spontaneously fused vesicles during 15 minutes and thus the turnover rate of a single spontaneously fusing vesicle (Additional file 1: Figure S2B). Using this single vesicle quantification, we determined the spontaneous turnover rate to be one vesicle each $80.42 \pm 10.77$ seconds, which is in accordance with literature [5]. Assuming a recycling pool size of about 130 vesicles [11-13] and neglecting the effect of reuse of vesicles and the faster recycling kinetics of the RRP vesicles [14], this fusion rate resulted in a rough estimate of about 174 minutes to turn over the whole recycling pool by spontaneous fusion, which agrees with the time course described above. Furthermore similar to evoked release [14] spontaneous vesicles were instantly re-releasable and thus immediately repopulate the readily releasable pool (Additional file 1: Figure S2).

In order to determine the strength of spontaneous release at the soma and the neuronal processes, we adopted a dual-color imaging approach with $\mathrm{spH}$ transfected neurons and an $\alpha$ GFP-CypHer5 $\mathrm{E}^{\mathrm{m}}$ antibody to label spontaneously fused synaptic vesicles (Figure 2A, B). Additionally we made use of a low transfection rate and chose neurons that fluoresced isolated among untransfected neighboring neurons for the analysis. The soma and the neurites were set manually (Figure $2 \mathrm{C}$ ). Sholl analysis revealed a normal branching pattern $\left(\Sigma_{\mathrm{spH}}=1.88 \pm\right.$ $0.34 ; \Sigma_{\text {mCherry }}=1.26 \pm 0.33$ ) [15]. We found that the size of the recycling pool as well as the number of spontaneously fused vesicles is higher at the soma than in distal cellular compartments indicating a higher absolute release. However, the relative amount of spontaneous turnover, normalized on the recycling pool size, was significantly higher in the periphery (Figure 2D). Therefore the smaller synapses in the periphery have a lower absolute spontaneous turnover, but release relative to their recycling pool more vesicles spontaneously. Distinction of the axon, the dendrites and the soma via MAP2 immunostaining also showed that in absolute values synapses near the soma released the highest number of spontaneous vesicles during a 120 minute period (Additional file 1: Figure S3). The fact that synapses located at the dendrite released more spontaneous vesicles compared to the axon can be explained by the shorter length of the dendrite and thus their closer average proximity to the soma. These results fit to the notion that vesicle recycling is more effective at smaller synapses [11] arguing for neuronal outgrowth in distal segments. Spontaneous release showed a strong correlation with the size of recycling and total pool at neurites and soma, respectively (Figure 2E, G). On the other hand, no clear relationship between spontaneous release and the reserve pool was observed (Figure 2F). The fact that spontaneous release correlates with the size of recycling and total pool, but not with the size of the reserve pool seems controversial. This can be due to the high variability of the pool sizes [16] especially the reserve pool [17] and heterogeneous release probabilities among synapses [18]. Similar to evoked release, the frequency distribution of spontaneous release is more right-skewed at the soma when compared to the process (Additional file 1: Figure S4A-C), which is consistent with more recycling vesicles at the soma-near synapses (Figure 2D). No differences between synapses at the dendrite and the axon could be observed (Additional file 1: Figure S4D). Synapses were then pooled and sorted into the $20 \%$ largest, smallest and the $20 \%$ around the medium size. 


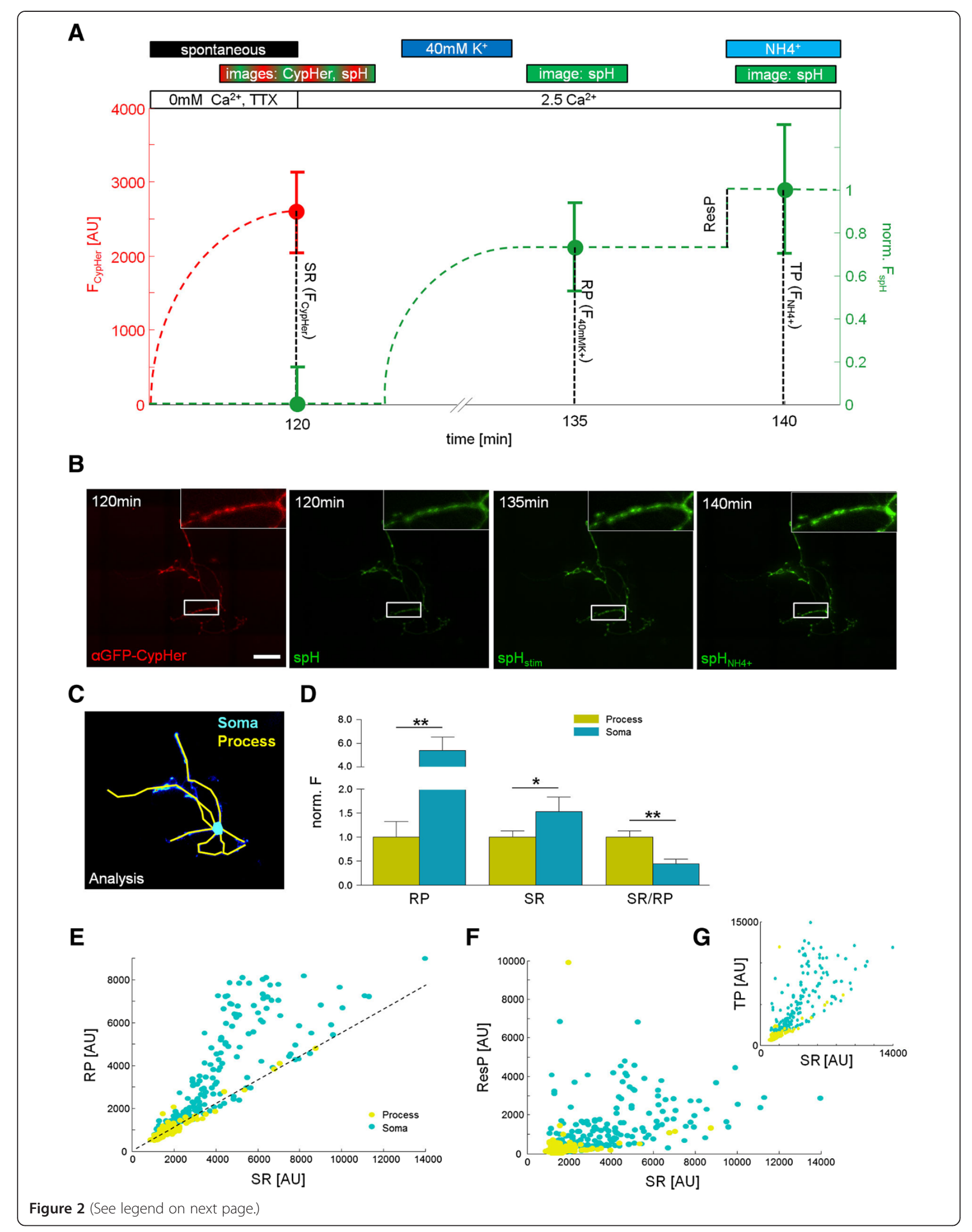


(See figure on previous page.)

Figure 2 At soma-near synapses the number of spontaneously fused vesicles as well the recycling pool vesicles is higher than in distal cellular compartments. A Analysis of spontaneous and evoked release differentiated between process and soma using dual color experiments of spH transfected hippocampal neurons spontaneously labeled with aGFP-CypHer5E ${ }^{\mathrm{Tm}}$. The spontaneous release (SR), recycling pool (RP), reserve pool (resP) and the total pool (TP) were determined. Scheme of experimental setup added with the mean values of spH and CypHer5E ${ }^{\mathrm{TM}}$

fluorescence used for further analysis. Boutons of spH transfected hippocampal neurons were first labeled with aGFP-CypHer5E ${ }^{\mathrm{TM}}$ by spontaneous uptake during a 120 minute period. Then a stitched image was captured to encompass the whole neuron with its processes at high resolution in both fluorescence channels (120 min). After stimulation with $40 \mathrm{mM} \mathrm{K}$ solution for 2 minutes in the presence of Bafilomycin an image of the same region was recorded (135 min), followed by an image after $\mathrm{NH}_{4}{ }^{+}$application (140 min). B Images depicting whole neuron. Insets: Synaptic boutons at higher magnification. Scale bar, $50 \mu \mathrm{m}$. C Scheme of image analysis used for distinction between process and soma. D Quantification of pool sizes: RP (two-sample $t$-test: $p=0.001$ ), SR (two-sample $t$-test: $p=0.042$ ) and SR to RP ratio (SR/RP; two-sample $t$-test: $p=0.005 ; 11$ experiments). Correlations of distinct vesicle pools differentiated for synapses located at the process or the soma: $\mathrm{SR}$ and RP (E; Pearson's $r_{\text {process }}=$ $\left.0.96 \pm 0.01, r_{\text {soma }}=0.84 \pm 0.04\right), S R$ and ResP (F; Pearson's $\left.r_{\text {process }}=0.19 \pm 0.14, r_{\text {soma }}=0.51 \pm 0.11\right)$ and SR and TP $\left(\mathbf{G} ;\right.$ Pearson's $r_{\text {process }}=0.64 \pm 0.09$, $\left.r_{\text {soma }}=0.78 \pm 0.05\right)$. Dashed line in $\mathbf{E}$ represents the linear fit of a subset of data putative resembling the processes running above or beneath the soma (slope $_{\text {process }}=0.55 \pm 0.02$, fit: $y=m x+t$ ).

Regarding these different synapse sizes and locations of individual boutons, these diverse distributions caused distinct fractions of the recycling and reserve pool size with respect to the total pool size (Additional file 1: Figure S4E). In absolute numbers spontaneous release declined with distance to the soma (Additional file 1: Figure S5A), but remained largely constant if spontaneous turn-over was normalized on synapse size (Additional file 1: Figure S5B).

\section{Conclusion and discussion}

We provide new evidence that spontaneous vesicle turnover can reach the level of the recycling pool of vesicles in a time and calcium dependent manner (Figure 1D, Additional file 1: Figure S1C). Considering these results together with the fact that the amount of recycling pool vesicles correlates robustly with the spontaneous release at each bouton (Figure 1E), suggest that spontaneous vesicles originate from the recycling pool of vesicles rather than from the reserve pool. This underlines previous findings $[5,6,19]$, but is contrary to studies that pointed at the reserve pool as origin of spontaneous vesicles [7]. These previous studies used a sequential labeling paradigm instead of a simultaneous labeling that we used in our study. We cannot exclude with our experimental approach that, after total recycling pool turnover, additional vesicles recycle spontaneously from within the reserve pool of vesicles, due to a lack of discrimination without sequential labeling after saturation. However such vesicles would account for a minority of spontaneously fusing vesicles due to the lack of correlation with the reserve pool (Figure 2F). Besides we found that the soma has absolutely the larger synapses with the larger recycling pool and the higher spontaneous release, but if spontaneous and evoked turnover is normalized on the size of the synapse, the relative release is higher at the processes. Recent publications found a distance from soma dependency of synapse size and evoked release at the processes [20,21]. In accordance with evoked release
[21], spontaneous release declined along the processes with increasing distance to the soma (Additional file 1: Figure S5A), but remained constant, if spontaneous release was normalized on synapse size (Additional file 1: Figure S5B). Our functional measurements indicated, that both forms of release exhibit the same relationship regarding distance from the soma with smaller, but more effective synapses at the process [11]. These results therefore point to a common developmental origin of these release modes with the vesicle populations stemming both from the recycling pool of vesicles. We also found differences between soma and processes regarding synapse size, relative and absolute release and confirmed, that smaller synapses release more efficiently. In conclusion we found a multitude of commonalities of spontaneous release and evoked release, e.g. correlation and identical size with recycling pool, vesicles were immediately re-releasable, same cellular localization with respect to release characteristics which together suggests the recycling pool as the common source of spontaneous and evoked released vesicles. Nevertheless a definition of a spontaneous vesicle pool is valid as vesicles differ with respect to their neuronal function and more future research is needed to substantiate the origin of spontaneous vesicles and to further address the function of these vesicles.

\section{Additional file}

Additional file 1: Figure S1. Validation of FM1-43 experiments using spH transfected neurons and an aGFP-CypHer5E ${ }^{\mathrm{TM}}$ antibody. Figure S2. Single vesicle release after spontaneous FM1-43 staining. Figure S3. Analysis of spontaneous release at the soma, the axon and the dendrite. Figure S4. Pool size distribution at the soma and the processes. Figure S5. Relationship between the distance from the soma and spontaneous release.

\section{Competing interests}

The authors declare no competing financial interests. 


\section{Authors' contributions}

$\mathrm{KL}$, OW designed and performed experiments. $\mathrm{KL}, \mathrm{OW}$ and $\mathrm{TWG}$ evaluated and wrote the manuscript. JK provided intellectual input. All authors read and approved the final manuscript.

\section{Acknowledgments}

We thank D. Gilbert and O. Friedrich (Institute of Medical Biotechnology, Friedrich-Alexander-University Erlangen-Nuremberg, Paul-Gordan-Strasse 3, Erlangen, Germany) for providing their imaging setup.

\section{Author details}

${ }^{1}$ Department of Psychiatry and Psychotherapy, Friedrich-Alexander-University of Erlangen-Nuremberg, Schwabachanlage 6, Erlangen 91054, Germany.

${ }^{2}$ Present address: Institute of Clinical Neuroimmunology, Ludwig-Maximilians University Munich, Munich, Germany.

Received: 8 July 2013 Accepted: 29 March 2014

Published: 2 April 2014

\section{References}

1. Emptage NJ, Reid CA, Fine A: Calcium stores in hippocampal synaptic boutons mediate short-term plasticity, store-operated Ca2+ entry, and spontaneous transmitter release. Neuron 2001, 29:197-208.

2. Autry AE, Adachi M, Nosyreva E, Na ES, Los MF, Cheng PF, Kavalali ET, Monteggia LM: NMDA receptor blockade at rest triggers rapid behavioural antidepressant responses. Nature 2011, 475:91-95

3. Sutton MA, Taylor AM, Ito HT, Pham A, Schuman EM: Postsynaptic decoding of neural activity: eEF2 as a biochemical sensor coupling miniature synaptic transmission to local protein synthesis. Neuron 2007, 55:648-661.

4. Sutton MA, Wall NR, Aakalu GN, Schuman EM: Regulation of dendritic protein synthesis by miniature synaptic events. Science 2004, 304:1979-1983.

5. Hua $Y$, Sinha R, Martineau M, Kahms M, Klingauf J: A common origin of synaptic vesicles undergoing evoked and spontaneous fusion. Nat Neurosci 2010, 13:1451-1453.

6. Groemer TW, Klingauf J: Synaptic vesicles recycling spontaneously and during activity belong to the same vesicle pool. Nat Neurosci 2007, 10:145-147.

7. Fredj NB, Burrone J: A resting pool of vesicles is responsible for spontaneous vesicle fusion at the synapse. Nat Neurosci 2009, 12:751-758.

8. Marra V, Burden JJ, Thorpe JR, Smith IT, Smith SL, Hausser M, Branco T, Staras K: A preferentially segregated recycling vesicle pool of limited size supports neurotransmission in native central synapses. Neuron 2012, 76:579-589.

9. Kavalali ET, Chung C, Khvotchev M, Leitz J, Nosyreva E, Raingo J, Ramirez DM: Spontaneous neurotransmission: an independent pathway for neuronal signaling? Physiology (Bethesda) 2011, 26:45-53.

10. Miesenbock G, De Angelis DA, Rothman JE: Visualizing secretion and synaptic transmission with $\mathrm{pH}$-sensitive green fluorescent proteins. Nature 1998, 394:192-195.

11. Welzel O, Henkel AW, Stroebel AM, Jung J, Tischbirek CH, Ebert K, Kornhuber J, Rizzoli SO, Groemer TW: Systematic heterogeneity of fractional vesicle pool sizes and release rates of hippocampal synapses. Biophys J 2011, 100:593-601.

12. Branco T, Staras K, Darcy KJ, Goda Y: Local dendritic activity sets release probability at hippocampal synapses. Neuron 2008, 59:475-485.

13. Ryan TA, Reuter H, Smith SJ: Optical detection of a quantal presynaptic membrane turnover. Nature 1997, 388:478-482.

14. Pyle JL, Kavalali ET, Piedras-Renteria ES, Tsien RW: Rapid reuse of readily releasable pool vesicles at hippocampal synapses. Neuron 2000, 28:221-231.

15. Sholl DA: Dendritic organization in the neurons of the visual and motor cortices of the cat. J Anat 1953, 87:387-406.

16. Moulder KL, Jiang X, Taylor AA, Shin W, Gillis KD, Mennerick S: Vesicle pool heterogeneity at hippocampal glutamate and GABA synapses. J Neurosci 2007, 27:9846-9854

17. Branco T, Marra V, Staras K: Examining size-strength relationships at hippocampal synapses using an ultrastructural measurement of synaptic release probability. J Struct Biol 2010, 172:203-210.
18. Murthy VN, Sejnowski TJ, Stevens CF: Heterogeneous release properties of visualized individual hippocampal synapses. Neuron 1997, 18:599-612.

19. Wilhelm BG, Groemer TW, Rizzoli SO: The same synaptic vesicles drive active and spontaneous release. Nat Neurosci 2010, 13:1454-1456.

20. Peng X, Parsons TD, Balice-Gordon RJ: Determinants of synaptic strength vary across an axon arbor. J Neurophysio/ 2012, 107:2430-2441.

21. de Jong AP, Schmitz SK, Toonen RF, Verhage M: Dendritic position is a major determinant of presynaptic strength. J Cell Biol 2012, 197:327-337.

doi:10.1186/1756-6606-7-23

Cite this article as: Loy et al:: Common strength and localization of spontaneous and evoked synaptic vesicle release sites. Molecular Brain 2014 7:23.

\section{Submit your next manuscript to BioMed Central and take full advantage of:}

- Convenient online submission

- Thorough peer review

- No space constraints or color figure charges

- Immediate publication on acceptance

- Inclusion in PubMed, CAS, Scopus and Google Scholar

- Research which is freely available for redistribution

Submit your manuscript at www.biomedcentral.com/submit
C) Biomed Central 